\title{
Post-launch radiometric validation of the GOES-16 Advanced Baseline Imager $(\mathrm{ABI})$
}

Jeffrey S. Czapla-Myers, Nikolaus J. Anderson

Jeffrey S. Czapla-Myers, Nikolaus J. Anderson, "Post-launch radiometric validation of the GOES-16 Advanced Baseline Imager (ABI)," Proc. SPIE 10785, Sensors, Systems, and Next-Generation Satellites XXII, 107851F (25 September 2018); doi: 10.1117/12.2324448

SPIE. Event: SPIE Remote Sensing, 2018, Berlin, Germany 


\title{
Post-launch radiometric validation of the GOES-16 Advanced Baseline Imager (ABI)
}

\author{
Jeffrey S. Czapla-Myers and Nikolaus J. Anderson \\ College of Optical Sciences, University of Arizona, 1630 E University Blvd, Tucson, AZ 85721-0094, USA
}

\begin{abstract}
The GOES-16 satellite was launched on 19 Nov 2016, and it became operational as the GOES-East satellite on 18 Dec 2017. The Advanced Baseline Imager (ABI) is one of six instruments onboard GOES-16. It has 16 spectral bands, a spatial resolution of $0.5-2.0 \mathrm{~km}$, and five times the temporal coverage of the previous GOES Imager. ABI has onboard radiometric calibration capabilities that were not available on the previous Imager instrument. The Radiometric Calibration Test Site (RadCaTS) is an automated facility composed of ground-based instruments that measure the surface reflectance and atmosphere throughout the day. It was developed by the Remote Sensing Group (RSG) of the College of Optical Sciences at the University of Arizona, and it is currently used to monitor such low-Earth orbit (LEO) sensors as Landsat-8 OLI, Terra and Aqua MODIS, Sentinel-2A and -2B MSI, SNPP VIIRS, and others. The successful launch of GOES-16, coupled with the improved spectral, spatial, and temporal characteristics of ABI, provide a unique opportunity to intercompare results obtained from a geosynchronous sensor to those obtained from typical LEO sensors. This work describes the recent efforts of RSG to validate the radiometric calibration of ABI, and compare the results with LEO sensors using RadCaTS.
\end{abstract}

Keywords: radiometric calibration, surface reflectance, GOES-16, ABI, RadCaTS

\section{INTRODUCTION}

GOES-R was launched on 19 Nov 2016, and renamed GOES-16 on 29 Nov 2016. ${ }^{1}$ After a one-year diagnostics and checkout phase, it was positioned in its final orbital position of $-75.2^{\circ}$ longitude (GOES East), and became operational on 18 Dec 2017. The GOES-16 platform contains six instruments, two of which are used for Earth observation purposes. The Geostationary Lightning Mapper (GLM) is a single-channel instrument that has one near infrared (NIR) channel to detect lightning events. ABI is the second Earth-observation instrument on GOES-16, and it is the primary imaging instrument. Its heritage originates from earlier GOES Imager instruments, but it has many improvements in its spatial, spectral, and calibration characteristics. ABI has 16 spectral bands that operate in the visible and near-infrared (VNIR), mid-wave infrared (MWIR), and the thermal infrared (TIR). The spatial resolution of ABI is also improved from previous Imager sensors, which makes it more amenable to ground-based in situ measurements for radiometric validation. The ABI calibration system consists of a solar diffuser for the solar-reflective bands, and an onboard blackbody for the emissive bands. Strategies for the post-launch validation of the ABI radiometric calibration have included an intensive field campaign in 2017 using a combination of in situ field measurements by ground personnel, an underflight with the Airborne Visible Infrared Imaging Spectrometer Next Generation (AVIRIS-NG) instrument onboard a high-altitude NASA ER-2 aircraft, and the intercomparison with Earth-observation satellite sensors. ${ }^{2-4}$

The Remote Sensing Group (RSG) of the College of Optical Sciences at the University of Arizona has developed an automated test site used for the validation of airborne and spaceborne sensors. ${ }^{5-7}$ The original prototype system used lightemitting diodes (LEDs) as detectors in three-channel VNIR radiometers that were used to measure the reflected radiance from the ground, and then converted to surface reflectance. ${ }^{8}$ One of the LED radiometers used during this prototype phase was pointed in the same viewing configuration as GOES I-M, and studies were performed to determine the feasibility of using such a ground-based system for the radiometric validation of an Earth-observation sensor in a geostationary orbit. ${ }^{9}$ The automated system was formalized as RadCaTS, and it now includes robust, thermally-stable, all weather groundviewing radiometers (GVRs), which eventually replaced the prototype LED radiometers in 2011-2012. ${ }^{10,11}$ The lessons learned using Railroad Valley for the radiometric calibration of the GOES I-M VNIR channel have been applied to this study, which summarizes the preliminary radiometric validation of the GOES-16 ABI solar-reflective channels, as well as the intercomparison of ABI with Terra and Aqua MODIS, Landsat-8 OLI, and Sentinel-2A and -2B MSI.

Sensors, Systems, and Next-Generation Satellites XXII, edited by Steven P. Neeck, Philippe Martimort, Toshiyoshi Kimura, Proc. of SPIE Vol. 10785, 107851F · @ 2018 SPIE · CCC code: 0277-786X/18/\$18 · doi: 10.1117/12.2324448 


\section{METHODOLOGY}

The RadCaTS algorithms for determining the surface reflectance, atmospheric transmission, and top-of-atmosphere (TOA) spectral radiance have been described in detail in other papers and will only be summarized here. ${ }^{12}$ The layout of the $1-\mathrm{km}^{2}$ region of interest at RadCaTS is shown in Fig. 1, which includes the position of the four nadir-viewing GVRs (21, 22, 24, and 25), the one GVR in the GOES-16 viewing configuration (23, Fig. 2), the meteorological station, Cimel solar lunar photometer ${ }^{13}$ and satellite uplink station. The multispectral GVRs make measurements every two minutes throughout the day when it is relatively cloud free, and the average surface reflectance is determined for each of the eight spectral channels. The average value is then fit to a library of $\sim 700$ hyperspectral reflectance spectra that were collected by RSG from 2000 2018. Atmospheric measurements are made using the Cimel CE318-T, which follows the NASA Aerosol Robotic Network (AERONET) protocol. The surface reflectance and atmospheric results are then used in the MODTRAN radiative transfer code to determine the hyperspectral TOA spectral radiance $(400-2500 \mathrm{~nm})$, which are band averaged to the sensor of interest. In the case of GOES-16 ABI, there is only one GVR that is used to make surface reflectance measurements. Work is currently underway to develop a bidirectional reflectance distribution function (BRDF) correction for near-nadir view angles $<15^{\circ}$, but the correction for an extreme view geometry such as GOES-16 has not been investigated at this point.
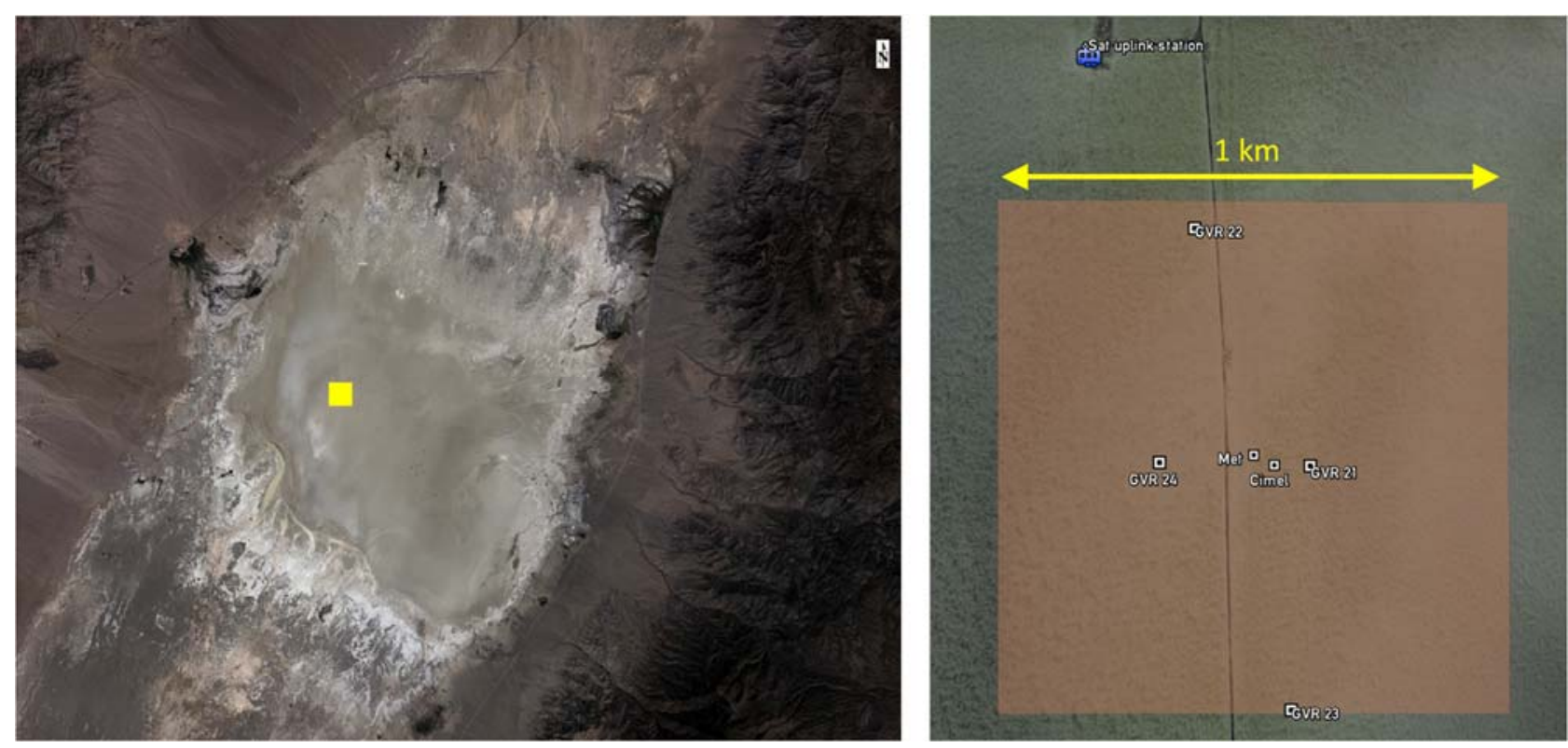

Fig. 1. Landsat-8 OLI image of Railroad Valley, Nevada. The RadCaTS 1-km² region of interest is shown as a yellow square (left). Details of the instrument locations within this area are shown on the right.

The intercomparison between GOES-16 ABI and LEO sensors is performed by choosing a suitable overpass date for the LEO sensor of interest. The temporal range of the comparison in this work is from Apr 2017 - Sep 2018. The overpass times and view geometries for Aqua, Terra, Landsat8, Sentinel-2A, and Sentinel-2B are shown in Table 1. A comparison of the spectral channels between each sensor and $\mathrm{ABI}$, including the spatial characteristics, is shown in Table 2. It should be noted that the ABI bands used in the comparison are limited to $1-3,5$, and 6 , since the signal-to-noise ratio of the RadCaTS measurements for ABI Band 4 (cirrus) is too low.

RadCaTS is one of four sites that currently make up the Committee on Earth Observation Satellites (CEOS) Working Group on Calibration and Validation (WGCV) Radiometric

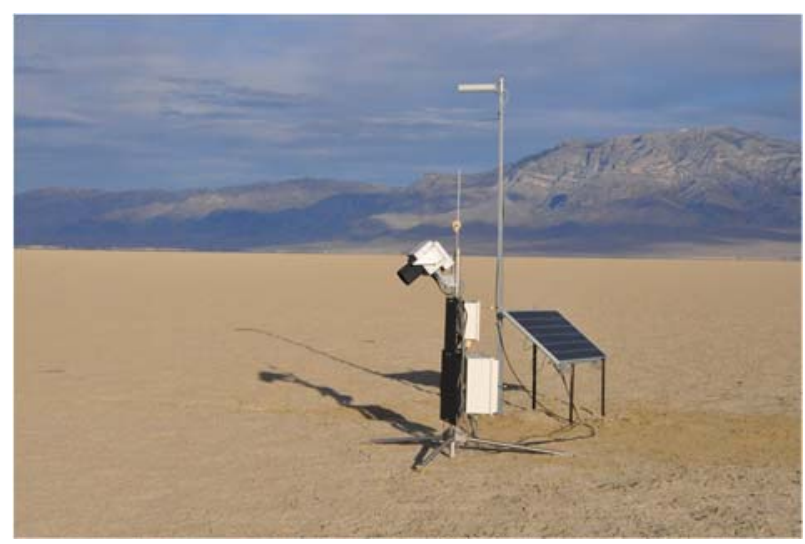

Fig. 2. GVR 23 in the GOES-16 viewing configuration. 
Calibration Network (RadCalNet, www.radcalnet.org). ${ }^{14-18}$ RadCalNet data are typically processed every 30 minutes from 09:00-15:00 local standard time (17:00-23:00 UTC at Railroad Valley), so an initial search in 2017 and 2018 began with dates that had a full set of RadCalNet data for a given day. This was done to identify days when the atmosphere was temporally stable throughout the day. Once this was completed, the candidate dates for ABI were matched with those of the LEO platforms. The comparison between LEO sensors and ABI are presented as the ratio of TOA spectral radiance (or reflectance) between the sensor of interest and RadCaTS.

\section{DATA}

The review of RadCalNet atmospheric results from Apr 2017 - Sep 2018 revealed 38 dates with atmosphericallystable conditions throughout the day, where all 13 individual times from 17:00-23:00 UTC had successful surface reflectance retrievals. In the case of the $\mathrm{ABI}$ radiometric validation using RadCaTS, solar noon was chosen to minimize the effects of shadowing on the ground. Railroad Valley is generally considered flat, but it can exhibit small topographic effects at the sampling scale of a GVR ( 27-cm spot size at nadir). An example of the surface reflectance of the RadCaTS 1- $\mathrm{km}^{2}$ region of interest as a function of time and wavelength during $4 \mathrm{Jul} 2017$ is shown in Fig. 3, where it should be noted that the reflectances are for a nadir-viewing configuration. The reflectance for ABI is determined by using the one GVR (Fig. 2) that is in the exact viewing configuration as ABI.
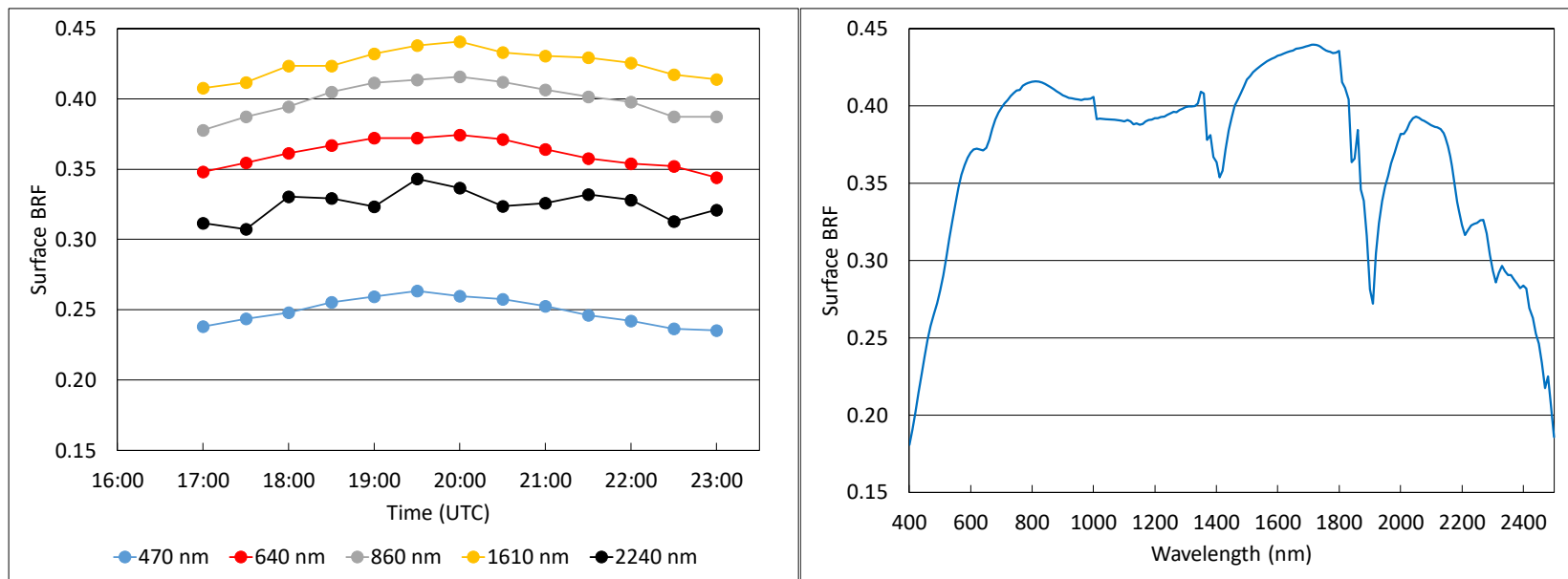

Fig. 3. Surface BRF for the RadCaTS 1- $\mathrm{km}^{2}$ region of interest on 4 Jul 2017. The surface bidirectional reflectance factor (BRF) for a nadir view is shown on the left for the five ABI bands, and the hyperspectral result for a nadir view at 20:30 UTC is shown on the right.

The ABI imagery was downloaded from the NOAA Comprehensive Large Array Stewardship System (CLASS) web portal, and an example of a Continental US (CONUS) image is shown in Fig. 4 for ABI Band $3(864 \mathrm{~nm})$ on $4 \mathrm{Jul}$ 2017. For each clear date, the at-sensor spectral radiance of channels $1-3,5$, and 6 was extracted for each of the CONUS images, and compared to the ground-based RadCaTS results. Once the radiometric comparison of ABI and RadCaTS was completed, the TOA results from each of the LEO sensors were summarized. Table 3 shows the period of study and the number of dates for each of the LEO sensors used in the intercomparison study.
Table 3. The period and number of dates for each LEO sensor in the ABI intercomparison.

\begin{tabular}{|ccc|}
\hline Sensor & Period & Number of Dates \\
TMODIS & $2014-2018$ & 45 \\
AMODIS & $2014-2018$ & 28 \\
S2A & $2015-2018$ & 34 \\
S2B & $2017-2018$ & 15 \\
L8 & $2013-2018$ & 23 \\
\hline
\end{tabular}




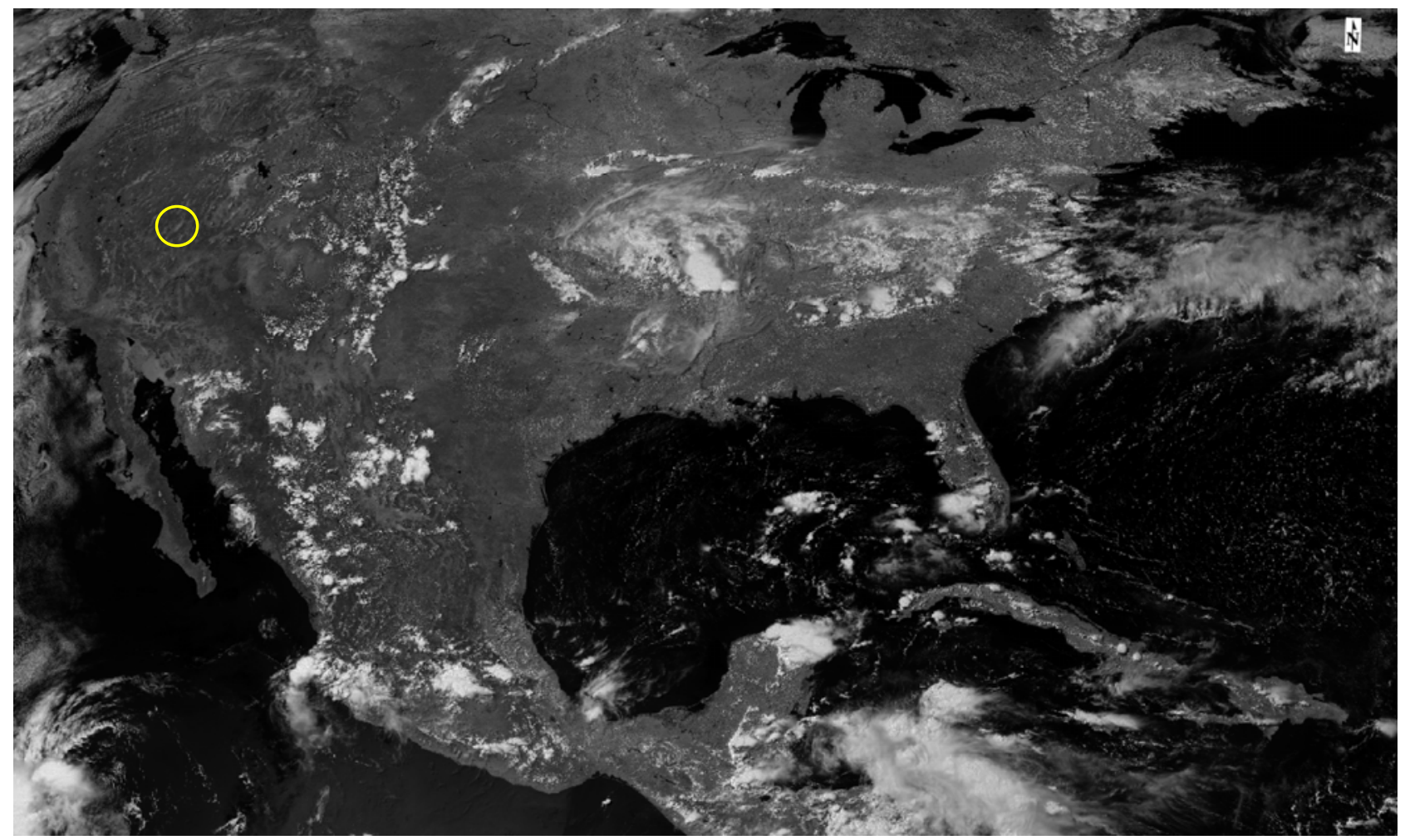

Fig. 4. A GOES-16 ABI Band 3 (864 nm) CONUS image from 4 Jul 2017 at 19:47 UTC. Railroad Valley is circled in yellow.

\section{RESULTS}

The radiometric validation results obtained using RadCaTS from Apr 2017 - Sep 2018 for GOES-16 ABI bands 1-3, 5, and 6 are shown in Fig. 5 for a solar noon collection time. The results are shown as the ratio of the TOA spectral radiance for each $\mathrm{ABI}$ band to the ground-based RadCaTS results, which have an estimated $\pm 4 \%$ uncertainty. The results in Fig. 5 include the 2017 average, the 2018 average, and the average of all data. The main reason for this separation is that the view geometry from GOES-16 to RadCaTS changed after moving to its final operational orbital location of $-75.2^{\circ}$ longitude, and the GVR used to measure the surface reflectance was repositioned to reflect this change.

The results for the radiometric validation of ABI indicate that in general, the reported atsensor spectral radiance is higher than those determined with RadCaTS, except for ABI Band $6(2243 \mathrm{~nm})$. The results from each of the two years in this study show agreement to within the uncertainties of RadCaTS. ABI Band 6 shows a larger change between years, which

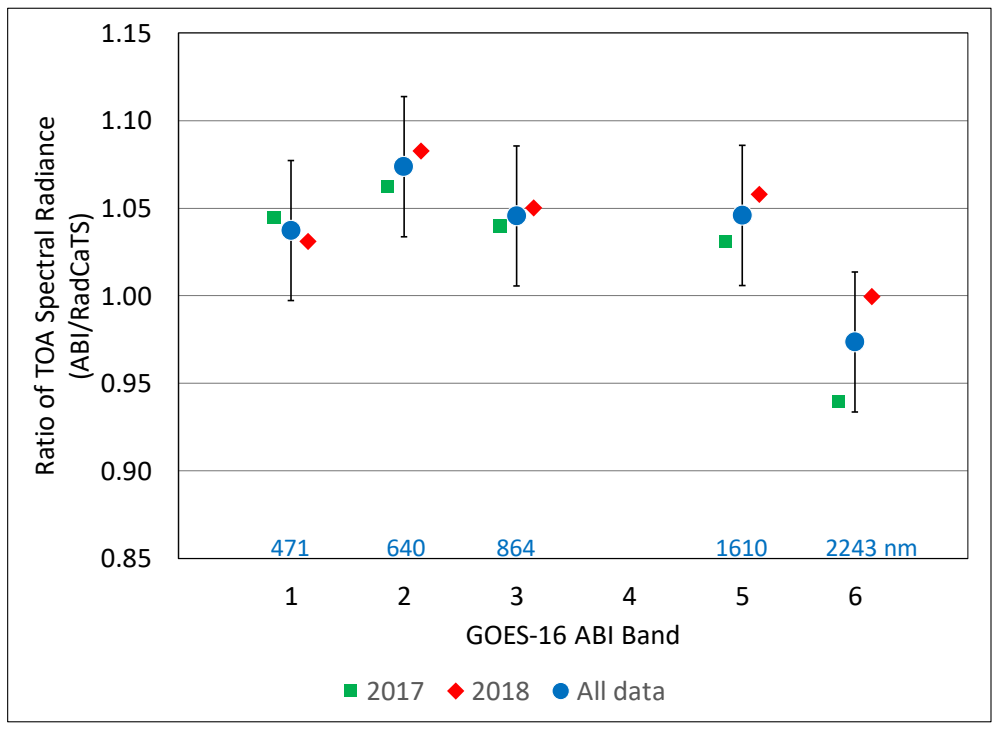

Fig. 5. A summary of the radiometric validation results of GOES-16 ABI using RadCaTS from Apr 2017 - Sep 2018. Results are shown as the 2017 average, 2018 average, and all data. The uncertainty bars are the $\pm 4 \%$ uncertainty estimate for RadCaTS (omitted for clarity for the individual years). The GOES16 view angle of RadCaTS was slightly different in 2017 (52.3 view zenith, $321.4^{\circ}$ view azimuth) and 2018 (61. $0^{\circ}$ view zenith, 306.1 ${ }^{\circ}$ view azimuth). The number of data points for each year is 17 in 2017 and 21 in 2018. 
is most likely due to the challenge of properly sampling the $2-\mathrm{km}$ pixel (which becomes a projected area of $\sim 4 \mathrm{~km}$ with the $61^{\circ}$ view angle) with a single radiometer.

The preliminary results from the comparison between ABI and similar bands for the LEO sensors used in this study are shown in Fig. 6. It should be noted that the results shown in Fig. 6 are based on the average values for each band of each sensor for a given timeframe, which is different for each sensor (Table 3). Therefore, no temporal trending is specified explicitly in the results provided here. The grouping of the results in Fig. 6 for each band shows that there appears to be a bias in ABI Bands 1, 2, and 6 when compared to LEO sensors.

\section{CONCLUSIONS}

This work summarizes the preliminary validation and intercomparison results for GOES-16 ABI bands 1-3, 5, and 6, using RadCaTS. Current results are limited to the

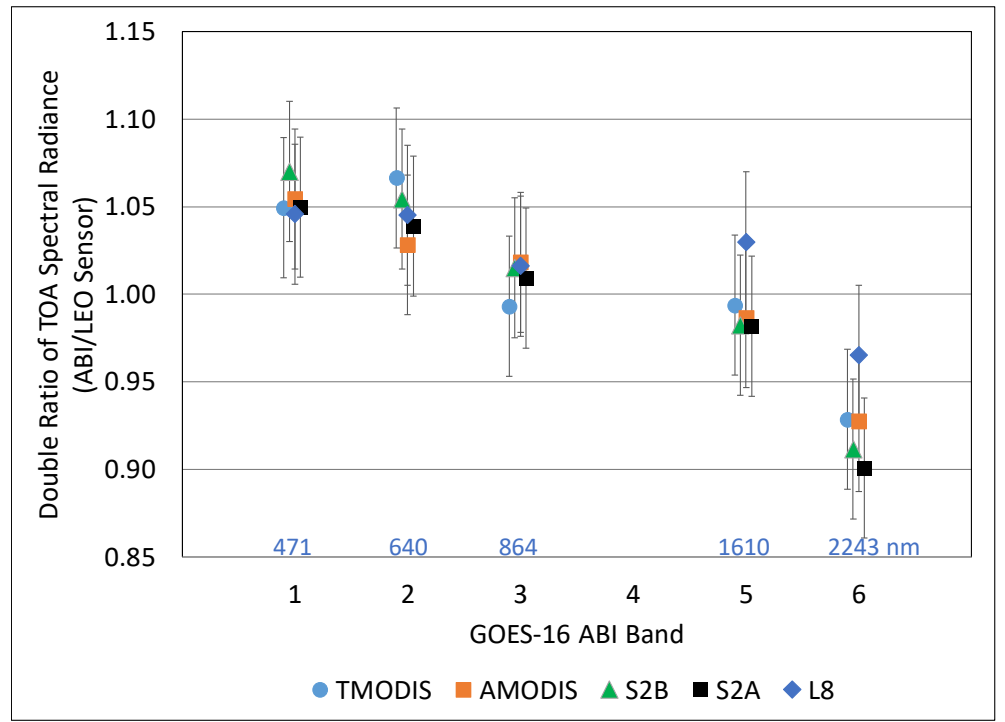

Fig. 6. The results of the intercomparison between GOES-16 ABI and the LEO sensors listed in Table 3, presented as a double ratio of the TOA spectral radiance: $(A B I /$ RadCaTS $) /(L E O /$ RadCaTS $)=A B I / L E O$. This effectively treats RadCaTS as a calibration transfer source and allows a direct comparison between $A B I$ and LEO sensors. measurements made at solar noon in an attempt to minimize the uncertainties due to shadowing effects on the surface. It was originally thought that the larger bias between ABI Band 6 and RadCaTS might be caused by a spatial sampling issue. There is only one GVR used to determine the surface reflectance for ABI. This issue is exasperated with the large view angle at Railroad Valley, but future work will incorporate studies to include data from the four nadir-viewing GVRs in an attempt to better sample the site. The main challenge will be converting the nadir-view surface reflectance to one corresponding to a $61^{\circ}$ view angle.

The intercomparison results between RadCaTS and the LEO sensors in this study show a similar bias between ABI and the LEO sensors, which suggests that the RadCaTS is spatially sampling the surface reflectance with reasonable accuracy for ABI work. The RadCaTS radiometric validation results also agree reasonably well with other ground-based measurements that were made in the Salar de Uyuni salt flats in Bolivia. ${ }^{19}$ The shape of the results from the Bolivian measurements as a function of wavelength are similar to those obtained with RadCaTS in this study, which increases the confidence that the bias observed with RadCaTS is real.

Future RadCaTS work with ABI will continue with daily data collection at Railroad Valley. The view zenith and azimuth angle of the GVR used to measure the surface reflectance has been updated to match the GOES East operational orbital position ( $-75.2^{\circ}$ longitude). Another GVR is being developed in anticipation of GOES-17 becoming operational as the GOES West satellite in 2018. Future comparisons between RadCaTS and ABI will include a more in-depth study of the radiometric validation throughout a given day. Results will be expanded from solar noon to determine if there are any temporal effects throughout the day, and this will also include studies of the RadCaTS surface reflectance retrieval. In addition to the radiometric validation work using RadCaTS, the intercomparison between ABI and LEO sensors will continue. Plans for future work will include creating a temporal comparison between ABI and LEO sensors, as opposed to using the average values over a given timeframe. This will allow any degradation in the ABI sensors to be monitored over time.

\section{ACKNOWLEDGEMENTS}

The authors would like to thank the Bureau of Land Management (BLM) Tonopah, Nevada, office for their assistance and permission in using Railroad Valley, and also AERONET for processing and distributing the Cimel data. The authors would also like to acknowledge NASA for funding this work through Research Grant NNX16AQ70G. 


\section{REFERENCES}

[1] T. J. Schmit, P. Griffith, M. M. Gunshor, J. M. Daniels, S. J. Goodman, and W. J. Lebair, "A Closer Look at the ABI on the GOES-R Series,” Bull. Amer. Meteor. Soc., 98(4), 681-698 (2017).

[2] F. Padula, A. J. Pearlman, C. Cao, and S. Goodman, "Towards post-launch validation of GOES-R ABI SI traceability with high-altitude aircraft, small near surface UAS, and satellite reference measurements." Proc. SPIE 9972, 14 (2016).

[3] J. P. Fulbright, E. Kline, D. Pogorzala, W. MacKenzie, R. Williams, K. Mozer, D. Carter, R. Race, J. Sims, and M. Seybold, "Calibration/validation strategy for GOES-R L1b data products." Proc. SPIE 10000, 11 (2016).

[4] F. Padula, S. J. Goodman, A. Pearlman, and C. Cao, "GOES-R Advanced Baseline Imager (ABI) and Geostationary Lightning Mapper (GLM) calibration/validation from a field campaign perspective." Proc. 2017 IEEE International Geoscience and Remote Sensing Symposium (IGARSS), 301-304 (2017).

[5] K. Thome, J. Czapla-Myers, N. Leisso, J. McCorkel, and J. Buchanan, "Intercomparison of Imaging Sensors using Automated Ground Measurements." Proc. IEEE IGARSS 4, IV - 1332-IV - 1335 (2008).

[6] K. J. Thome, J. S. Czapla-Myers, and K. Scott, "Automated ground system for reflectance-based calibration." Proc. CALCON Technical Conference, 1-13 (2005).

[7] J. Czapla-Myers, K. Thome, and S. Biggar, "Unmanned vicarious calibration for large-footprint sensors." Proc. SPIE 5882, 588218-10 (2005).

[8] J. S. Czapla-Myers, K. J. Thome, and S. F. Biggar, "Design, calibration, and characterization of a field radiometer using light-emitting diodes as detectors," Applied Optics, 47(36), 6753-62 (2008).

[9] N. P. Leisso, K. J. Thome, and J. S. Czapla-Myers, "Validation of the onboard radiometric calibration of the GOES I-M visible channel by reflectance-based vicarious methods." Proc. SPIE 6684, 668404-10 (2007).

[10] N. J. Anderson, and J. S. Czapla-Myers, "Ground viewing radiometer characterization, implementation and calibration applications: a summary after two years of field deployment." Proc. SPIE 8866, 88660N-88660N-10 (2013).

[11] N. Anderson, J. Czapla-Myers, N. Leisso, S. Biggar, C. Burkhart, R. Kingston, and K. Thome, "Design and calibration of field deployable ground-viewing radiometers," Appl. Opt., 52(2), 231-240 (2013).

[12] J. Czapla-Myers, J. McCorkel, N. Anderson, K. Thome, S. Biggar, D. Helder, D. Aaron, L. Leigh, and N. Mishra, "The Ground-Based Absolute Radiometric Calibration of Landsat 8 OLI," Remote Sensing, 7(1), 600-626 (2015).

[13] Á. Barreto, E. Cuevas, M. J. Granados-Muñoz, L. Alados-Arboledas, P. M. Romero, J. Gröbner, N. Kouremeti, A. F. Almansa, T. Stone, C. Toledano, R. Román, M. Sorokin, B. Holben, M. Canini, and M. Yela, "The new sun-sky-lunar Cimel CE318-T multiband photometer - a comprehensive performance evaluation," Atmos. Meas. Tech., 9(2), 631-654 (2016).

[14] A. Bialek, C. Greenwell, M. Lamare, A. Meygret, S. Marcq, S. Lachérade, E. Woolliams, B. Berthelot, M. Bouvet, M. King, C. Underwood, and N. Fox, "New radiometric calibration site located at Gobabeb, Namib desert." Proc. 2016 IEEE International Geoscience and Remote Sensing Symposium (IGARSS), 6094-6097 (2016).

[15] C. Ong, A. Mueller, K. Thome, M. Bachmann, J. Czapla-Myers, S. Holzwarth, S. J. Khalsa, C. MacLellan, T. Malthus, J. Nightingale, L. Pierce, and H. Yamamoto, "Report on International Spaceborne Imaging Spectroscopy Technical Committee calibration and validation workshop, national environment research council field spectroscopy facility, University of Edinburgh." Proc. 2016 IEEE International Geoscience and Remote Sensing Symposium (IGARSS), 1909-1911 (2016).

[16] T. Scanlon, C. Greenwell, J. Czapla-Myers, N. Anderson, T. Goodman, K. Thome, E. Wolliams, G. Porrovecchio, P. Linduška, M. Šmíd, and N. P. Fox, "Ground comparisons at RadCalNet sites to determine the equivalence of sites within the network." Proc. SPIE 10423, 13 (2017).

[17] A. C. Banks, S. E. Hunt, J. Gorroño, T. Scanlon, E. R. Woolliams, and N. P. Fox, "A comparison of validation and vicarious calibration of high and medium resolution satellite-borne sensors using RadCalNet." Proc. SPIE 10423, 9 (2017).

[18] N. Wang, C. Li, L. Ma, Y. Liu, F. Meng, Y. Zhao, B. Pang, Y. Qian, W. Li, L. Tang, and D. Wang, "Groundbased automated radiometric calibration system in Baotou site, China." Proc. SPIE 10427, 8 (2017).

[19] J. McCorkel, Personal communication with author, Sep, 2018. 